\title{
Progressive hearing loss in Fabry's disease: a case report
}

\begin{abstract}
Fabry's disease is a chromosomal X-linked inherited disease, which causes a lack of the lysosomal alpha-galactosidase A enzyme leading to a cellular accumulation of glycosphingolipids. This accumulation leads to various clinical disorders, including inner ear lesions, with sensorineural hearing loss and dizziness. This article proposes to describe a clinical case of a patient suffering from Fabry's disease with inner ear associated problems and to review the literature focusing on this subject.
\end{abstract}

Keywords Fabry's disease - Inner ear · Hearing loss · Sudden deafness

\section{Introduction}

Fabry's disease is a chromosomal X-linked inherited disorder that causes the lack of a lysosomal hydrolase (alpha-galactosidase A), leading to an alteration of the metabolism and a progressive accumulation of glycosphingolipids (predominantly the globotriosylcercamides), into the lysosomes of the whole organism's cells and into the blood plasma. The oto-rhino-laryngological area is not spared by this disease, and the majority of patients develop a progressive and accelerated sensorineural hearing loss during adulthood. One third of the patients may undergo a sudden unilateral sensorineural hearing loss. In addition, a high incidence of vestibular disorders with dizziness and chronic instability is also observed in these patients.

This article describes a case of a patient affected by Fabry's disease that included both a progressive bilateral sensorineural hearing loss and an additional sudden

F. M. Barras $(\bowtie) \cdot$ R. Maire

Department of Oto-Rhino-Laryngology and Cervico-Facial

Surgery, Lausanne University Hospital (CHUV), 1011,

Lausanne, Switzerland

E-mail: florian.barras@chuv.hospvd.ch

Tel.: $+41-21-3142676$

Fax: + 41-21-3142646 unilateral hearing loss. The case report is followed by a review of the literature concerning the neurotological consequences of Fabry's disease.

\section{Case report}

A 60-years-old Caucasian male patient was positive for Fabry's disease and presented the majority of its manifestations: acrodynia, acroparesthesia, angiokeratoma, vasculitis, hypertrophic cardiomyopathy complicated by an atrio-ventricular blockage (a pace maker was implanted in January 2003), a discrete mitral insufficiency, and terminal renal insufficiency that required a renal graft in March, 2003. Before transplantation, the patient was treated with haemodialysis three times per week since December 2001. The post-transplantation treatment was realized with an association of Cyclosporine (100 mg-0-75 mg) and Tacrilomus ( $1 \mathrm{~g}-0-1 \mathrm{~g})$, and was no modified later.

The patient was referred for an audiological consultation in the beginning of April 2003 to evaluate the necessity of a hearing aid. He complained of a bilateral progressive hearing loss associated with tinnitus, leading to social and professional conversational disturbances. Examination revealed normal tympanic membranes, a midline Weber test and a positive Rinné test on both sides. Pure-tone and speech audiometry were performed. There was a moderate sensorineural hearing loss bilaterally, with poorer sensitivity on the left side (Fig. 1a). The pure tone average (PTA) between $0.5-4 \mathrm{kHz}$ was $37 \mathrm{~dB}$ HL on the right side and $55 \mathrm{~dB}$ HL on the left side. Speech understanding ability was evaluated with monosyllabic words and revealed a speech recognition threshold of $72 \mathrm{~dB}$ at the right ear and $100 \mathrm{~dB}$ at the left ear (Fig. 1b). According to these results, the patient has been rehabilitated stereophonically.

At the beginning of May 2003, the patient experienced a period of fever that lasted for 1 week. He was bed ridden because of the fever and increased dizziness. During this period, he did not use his hearing aids. One 


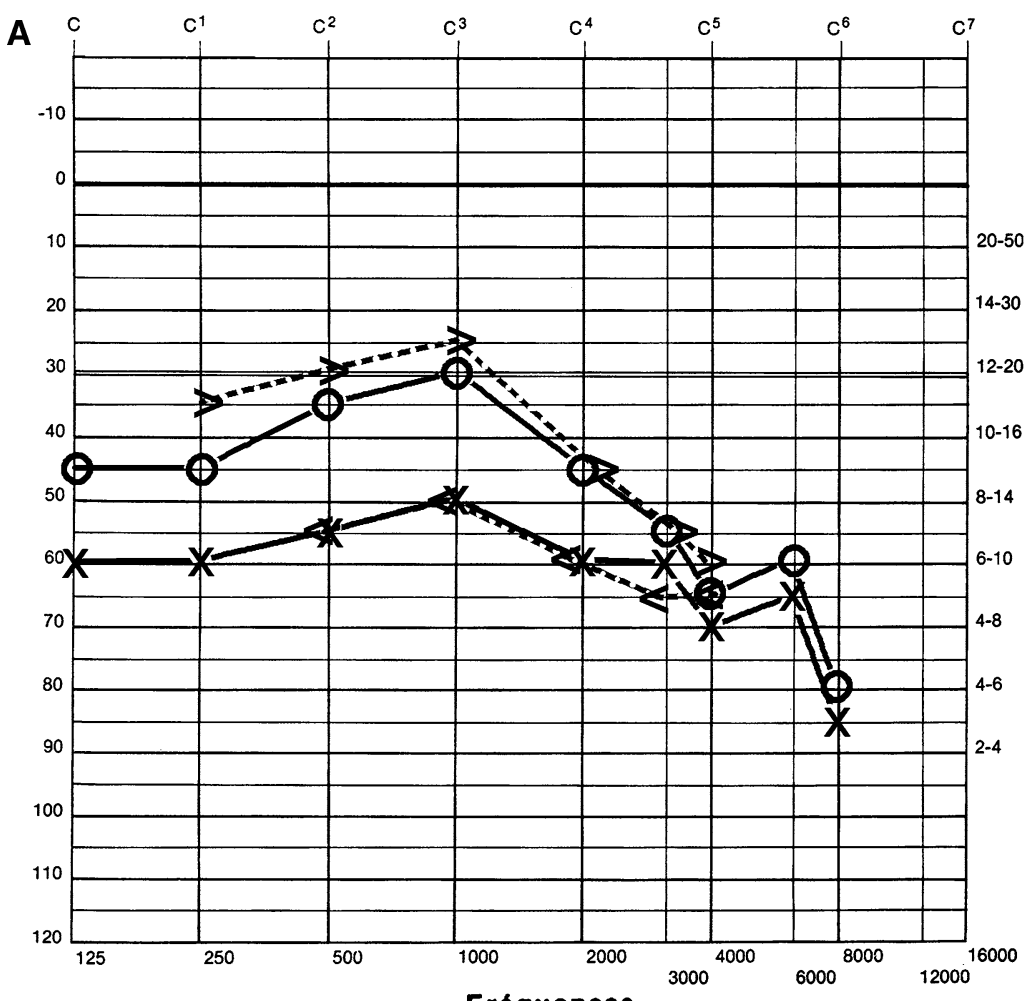

B

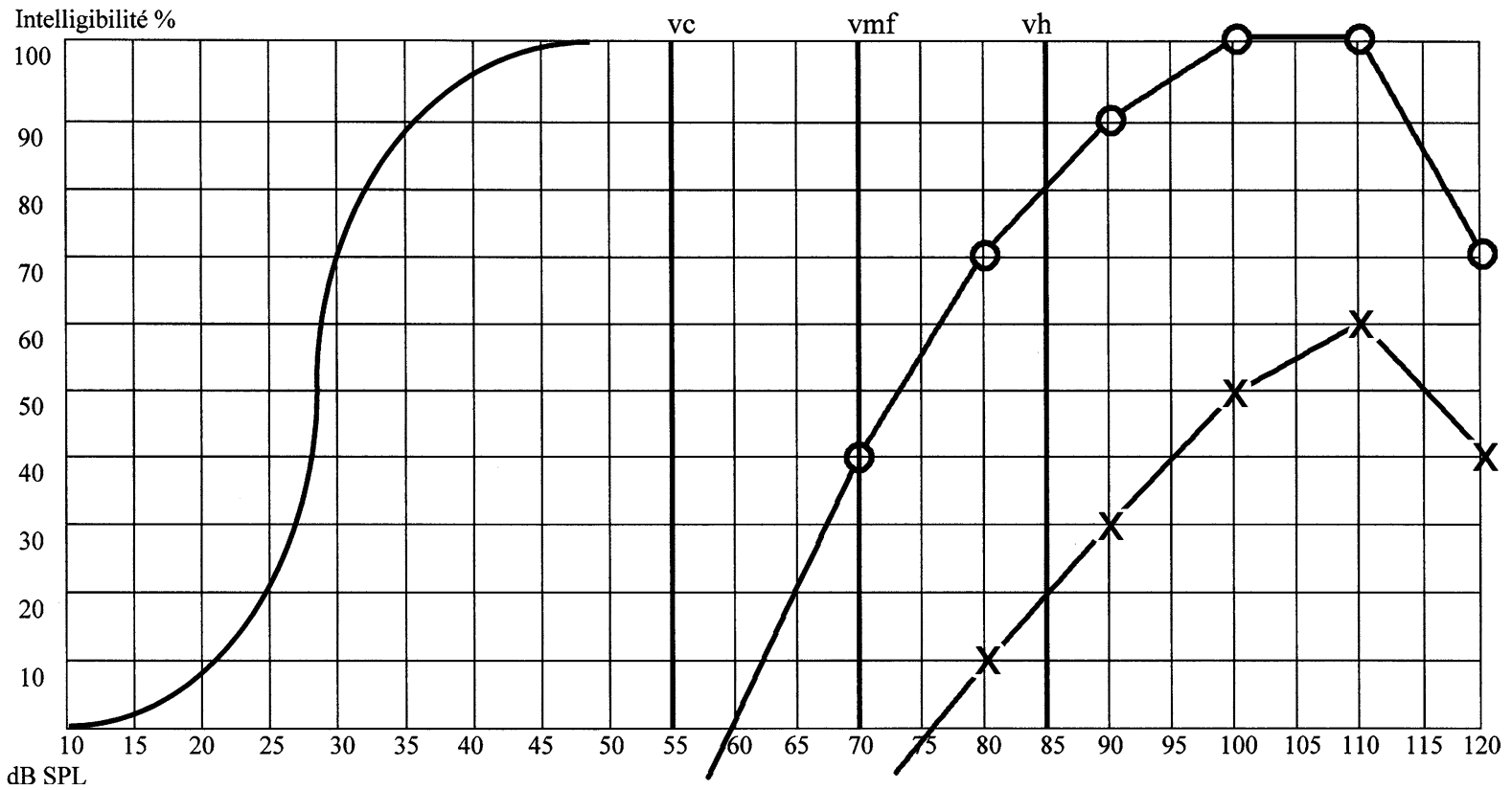

Fig. 1 Audiological evaluation in April 2003. a Pure tone audiogram: pure tone average (PTA) between $0.5-4 \mathrm{KHz}$ of $37 \mathrm{~dB}$ HL on the right (circle) side and of $55 \mathrm{~dB}$ HL on the left

week later, he tried to use his hearing aids and noticed that he could not hear on the left side. The patient presented for audiological consultation the 23rd of May 2003. The clinical examination showed a normal tympanic membrane on both sides. The Weber test showed a lateralization to the right side, while the Rinné was positive on the right side and the stimulus could not side (cross). b Speech audiogram (monosyllables): speech recognition threshold of $72 \mathrm{~dB}$ on the right ear (circle) and $100 \mathrm{~dB}$ at the left ear (cross)

be perceived on the left side. The vestibular examination yielded the following results: normal function of cranial nerves (excepted for the VIIIth cranial pair), wellperformed cerebellar tests, normal Romberg and Unterberger tests, normal smooth pursuit and complete inhibition of perotatory nystagmus with ocular fixation. The examination under videoscopy showed no 
spontaneous nystagmus, no positional (-ing) nystagmus, and no nystagmus after head shaking. However, the bi-thermal binaural caloric tests showed a complete areflexy on both sides. The examination was completed with a pure tone audiogram, which confirmed a complete loss of hearing sensitivity on the left side, with test results for the right side similar to those obtained in April 2003 (Fig. 2). The electroacoustic characteristics of the hearing aid for the right side were adjusted to reflect the change from a binaural to a monaural fitting, and hearing aid use for the left side was discontinued.

During July 2003, the patient experienced a further deterioration of the hearing sensitivity on the right ear, with an additional $20 \mathrm{~dB}$ loss at $500 \mathrm{~Hz}$, and $10 \mathrm{~dB}$ at the other frequencies (Fig. 2). A corticotherapy with $1 \mathrm{mg} / \mathrm{kg}$ prednisone during 10 days was administered, but no significant recovery was observed.

Audiometric evaluation was preformed at the end of September 2003, and the hearing sensitivity for the right ear was unchanged from results obtained on July 2003. No recovery occurred on the left side. A BICROS system with amplification of the right ear was proposed to the patient, but he preferred to retain the monaural fitting.

\section{Discussion and review of the literature}

Fabry's disease is a rare genetic disorder, with a frequency of occurrence of 1 in 100,000 births [1]. It is the second most common glycosphingolipid storage disorder after Gaucher's disease. Considering the inner ear, Fabry's disease frequently leads to labyrinth dysfunctions, such as sensorineural hearing loss, sudden deafness, tinnitus and dizziness or vertigo[2, 3, 4, 5]. A sensorineural hearing loss is the most common manifestation of the disease in the labyrinth. As many as $50-78 \%$ of patients affected with Fabry's disease will develop auditory symptoms during their life, as observed in two studies including 22 and 98 patients with Fabry's disease, respectively [2, 3]. An increased incidence of tinnitus and sudden sensorineural hearing loss has also been reported in Fabry's disease compared to the normal population [2]. This situation was encountered in our patient, who first experienced a progressive hearing loss on both ears, followed by a sudden hearing loss on the left side.

The causes of hearing loss (progressive or sudden) in patients affected by Fabry's disease are still not known. Some authors postulate that it is not different from the presbycusis present in the normal population [5]. However, some hypotheses to explain both the progressive and sudden sensorineural hearing loss have been reported in the literature: deposition of glycosphingolipids into the inner ear [2], long term obstruction of cranial vessels by dolicho-ectatic arteries, due to the local deposition of glycosphingolipids into the wall of arteries [4]. Postmortem histopathologic examination of temporal bones of affected patients have shown both middle and inner ear alterations: seropurulent effusions
Fig. 2 Audiological evaluation since May 2003. Left ear: complete deafness, no further recovery. Right ear: degree of pure tone thresholds in May $(A)$, and on retest in July and September $(B)$

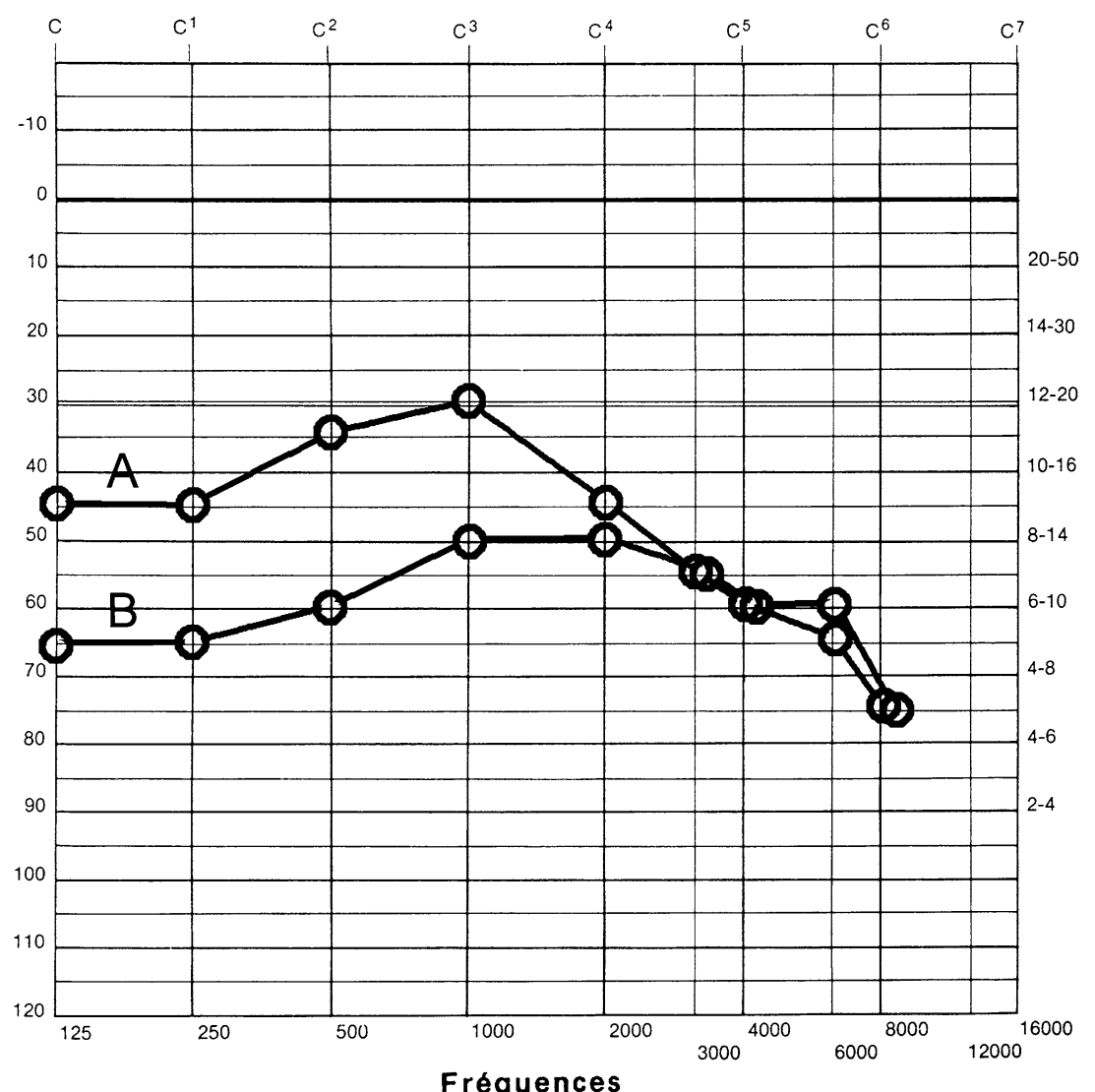

Fréquences 
and hyperplastic mucosa were found in middle ears; strial and spiral ligament atrophy, hair cell loss and reduced number of fibers in spiral ganglia were found in inner ears; no evidence of glycosphingolipid accumulation was observed in spiral ganglia [6]. Other authors postulate that the hearing loss could be attributed to chronic renal insufficiency and dialysis, which have been described as potentially deleterious for the inner ear[7, 8]. Fabry's disease is also known for its impact on the balance system, leading to dizziness. The major cause of dizziness is an alteration of the central balance centers (cerebellar vermis and vestibular nuclei) occasioned by focal infarcts due to thrombotic zones into these central balance centers [4]. However, a peripheral origin can also be detected with bilateral areflexia after caloric stimulation [2], as found in our patient.

Initial signs of Fabry's disease can be detected during infancy. They include angiokeratoma, acroparesthesia, acrodynia, dysautonomia and corneal opacities. Further deposition of glycosphingolipids into the endothelial cells and in the smooth muscle cells of micro-vessels leads to narrowing and thrombosis of little arteries and arterioles. These depositions may produce ischemic cerebral and cardiac complication as well as progressive renal insufficiency, which may lead to premature death. However, the disease can be recognized during childhood by specific neurological symptoms such as severe episodes of lancinating pain or burning paresthesia in the extremities [9]. The early detection of the disease during infancy is important to initiate treatment as early as possible. Indeed, enzyme replacement therapy is emerging as a new treatment, with good results. It allows the accumulation of glycosphingolipids into the wall of small arteries to be delayed or even stopped, thus preventing the life-threatening consequences of the disease $[9,10,11,12]$.

\section{References}

1. Meikle PJ, Hopwood JJ, Clague AE, Carey MF (1999) Prevalence of lysosomal storage disorders. Jama 281:49-54

2. Germain DP, Avan P, Chassaing A, Bonfils P (2002) Patients affected with Fabry disease have an increased incidence of progressive hearing loss and sudden deafness: an investigation of twenty-two hemizygous male patients. BMC Med Genet 3:10

3. MacDermot KD, Holmes A, Miners AH (2001) AndersonFabry disease: clinical manifestations and impact of disease in a cohort of 98 hemizygous males. J Med Genet 38:750-760

4. Mitsias P, Levine SR (1996) Cerebrovascular complications of Fabry's disease. Ann Neurol 40:8-17

5. Morgan SH, Rudge P, Smith SJ, Bronstein AM, Kendall BE, Holly E, Young EP, Crawfurd MD, Bannister R (1990) The neurological complications of Anderson-Fabry disease (alphagalactosidase A deficiency)-investigation of symptomatic and presymptomatic patients. Q J Med 75:491-507

6. Schachern PA, Shea DA, Paparella MM, Yoon TH (1989) Otologic histopathology of Fabry's disease. Ann Otol Rhinol Laryngol 98:359-363

7. Ozturan O, Lam S (1998) The effect of hemodialysis on hearing using pure-tone audiometry and distortion-product otoacoustic emissions. ORL J Otorhinolaryngol Relat Spec 60:306-313

8. Stavroulaki P, Nikolopoulos TP, Psarommatis I, Apostolopoulos N (2001) Hearing evaluation with distortion-product otoacoustic emissions in young patients undergoing haemodialysis. Clin Otolaryngol 26:235-242

9. Hilz MJ (2002) Evaluation of peripheral and autonomic nerve function in Fabry disease. Acta Paediatr Suppl 91:38-42

10. Breunig F, Wanner C (2003) Enzyme replacement therapy for Fabry disease: proving the clinical benefit. Nephrol Dial Transplant 18:7-9

11. Breunig F, Weidemann F, Beer M, Eggert A, Krane V, Spindler M, Sandstede J, Strotmann J, Wanner C (2003) Fabry disease: diagnosis and treatment. Kidney Int Suppl 84:181-185

12. Desnick RJ, Brady R, Barranger J, Collins AJ, Germain DP, Goldman M, Grabowski G, Packman S, Wilcox WR (2003) Fabry disease, an under-recognized multisystemic disorder: expert recommendations for diagnosis, management, and enzyme replacement therapy. Ann Intern Med 138:338-346 